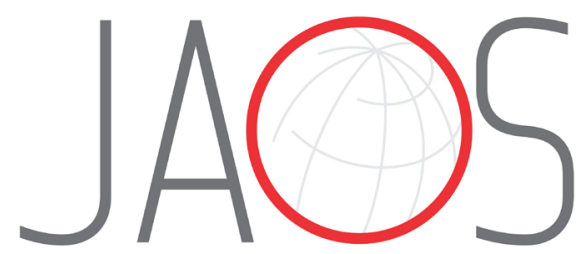

JOURNAL OF APPLIED ORAL SCIENCE

\title{
A comparison of the sealing abilities between Biodentine and MTA as root-end filling materials and their effects on bone healing in dogs after periradicular surgery
}

\section{Abstract}

Jing-jing TANG ${ }^{1,2 *}$

Zong-shan SHEN ${ }^{1 *}$

Wei QIN ${ }^{1}$

Zhengmei LIN ${ }^{1}$
Submitted: December 05, 2018 Modification: January 24, 2019 Accepted: March 27, 2019

Corresponding address: Zhengmei Lin 56 Linyuan West Road - Guangzhou - China. e-mail: linzhm@mail.sysu.edu.cn
Objectives: To compare the sealing ability and biocompatibility of Biodentine with mineral trioxide aggregate (MTA) when used as root-end filling materials. Methodology: The Cell Counting Kit-8 (CCK-8) assay was used to compare the cytotoxicity of MTA and Biodentine. Twenty-one extracted teeth with a single canal were immersed in an acidic silver nitrate solution after root-end filling. Then, the volume and depth of silver nitrate that infiltrated the apical portion of the teeth were analyzed using micro-computed tomography (micro-CT). Seventy-two roots from 3 female beagle dogs were randomly distributed into 3 groups and apical surgery was performed. After six months, the volume of the bone defect surrounding these roots was analyzed using micro-CT. Results: Based on the results of the CCK- 8 assay, MTA and Biodentine did not show statistically significant differences in cytotoxicity $(P>0.05)$. The volume and the depth of the infiltrated nitrate solution were greater in the MTA group than in the Biodentine group $(P<0.05)$. The volume of the bone defect was larger in the MTA group than in the Biodentine group. However, the difference was not significant $(P>0.05)$. The volumes of the bone defects in the MTA and Biodentine groups were smaller than the group without any filling materials $(P<0.05)$. Conclusions: MTA and Biodentine exhibited comparable cellular biocompatibility. Biodentine showed a superior sealing ability to MTA in root-end filling. Both Biodentine and MTA promoted periradicular bone healing in beagle dog periradicular surgery models.

Keywords: MTA. Biodentine. Periradicular surgery. Biocompatibility. Sealing ability 


\section{Introduction}

Root canal treatment is generally performed to treat dental pulp and periapical disease. When the root canal treatment and retreatment fail, periradicular surgery is the last hope for the affected teeth. ${ }^{1}$ The aim of periradicular surgery is not simply to remove infected apical tissue or the root tip, but most importantly is to reseal the root canal system. ${ }^{2}$ For this purpose, a root-end filling material must be applied to fill the root-end cavity and seal the exposed dentine during periradicular surgery. Hence, this material must possess sealing ability and biocompatibility. In addition, it should have antibacterial qualities and be easy to manipulate. ${ }^{3}$

Mineral trioxide aggregate (MTA), due to its superior characteristics compared to other traditional root-end filling materials such as Amalgam, guttapercha and glass ionomer cement, ${ }^{4}$ is one of the most ideal root-end filling materials. It is also indicated in direct capping, apexification and perforation repair in dentistry. Its good biocompatibility and sealing ability have been verified in long-term clinical practice since the mid-1990s. ${ }^{5}$ However, the drawbacks of MTA, such as the potential for tooth discoloration, difficult handling characteristics and long setting time, limit its applications. , $6^{6}$ Current research is actively seeking alternative materials to MTA.

Biodentine is a new tricalcium silicate-based cement material that was introduced in 2009. It has better handling properties and a shorter setting time than MTA. In addition, it possesses very similar physical properties to dentine and poses low risk of tooth discoloration. The powder mainly consists of tricalcium silicate, zirconium, and calcium carbonate. The liquid is composed of water, calcium chloride and hydrosoluble polymer. $^{7}$

The Biodentine manufacturer claims that its applications are similar to MTA. For example, as a direct capping agent, Biodentine produces good results in asymptomatic vital permanent teeth with cariously exposed pulp. ${ }^{8-11}$ Moreover, it has also been applied in pulpotomy and apexification. ${ }^{12,13}$ Actually, the induction of mineralization of this bioactive material has been verified at the cell and molecular levels. ${ }^{14}$ As shown in the study by Daltoe, Biodentine induces similar levels of mineralization markers in the pulp compared with MTA. Together, animal models in which Biodentine has been employed in furcation perforation repair show that both Biodentine and MTA are excellent perforation repair materials, but MTA results in a greater frequency in complete sealing of the furcation perforation. ${ }^{15}$ However, the effect of Biodentine on the periapical tissue has not been completely elucidated. The apical circumstance is more complex than tooth furcation and root canals. Hence, the sealing ability and biocompatibility of Biodentine as a root-end filling material must be studied. This study aims to compare citocompatibility in human periodontal ligament cells and sealing ability of Biodentine to MTA when used as root-end filling materials and their effects on apical bone healing after periapical surgery in dogs.

\section{Methodology}

\section{Cell Culture and Material Preparation}

Three healthy, impacted third molars were extracted from three healthy male patients aged 18-22 years old. Informed consent was obtained from all patients before extraction. Briefly, the periodontal ligament was cut into pieces and digested in a solution of $2 \mathrm{mg} / \mathrm{mL}$ collagenase I (Invitrogen-Life Technologies, CA, USA) for 20 minutes at $37^{\circ} \mathrm{C}$. The cells were centrifuged and resuspended. The cell suspension was seeded into a T-25 flask (Costar, Cambridge, USA) containing a-MEM supplemented with $20 \%$ FBS, $2 \%$ penicillin and streptomycin. Then, the cells were incubated in a $5 \%$ carbon dioxide incubator (Shellab, Cornelious, OR, USA) at $37^{\circ} \mathrm{C}$. The cells were sub-cultured after reaching $80-85 \%$ confluence, and cells at the third passage were used on subsequent experiments. Periodontal ligament cells (PDLCs) were cultured in media supplemented with $10 \%$ FBS.

Both Biodentine (Septodont, Saint Maur des Fosses, France) and Pro $\square$ Root MTA (Dentsplay, Tulsa, USA) were prepared and set under aseptic conditions according to the manufacturer's instructions. After setting, $0.2 \mathrm{~g}$ of MTA or Biodentine was immersed in $1 \mathrm{~mL}$ of a-MEM supplemented with $10 \%$ FBS and incubated for $72 \mathrm{~h}$ at $37^{\circ} \mathrm{C}$. Then, the materials were discarded and the eluate extracts were filtered with 0.22- $\mu \mathrm{m}$ pore size membranes (Millipore; Billerica, MA, USA ). ${ }^{16,17}$

\section{Cell Counting Kit-8 (CCK-8) Assay}

One hundred microliters of suspended PDLCs were dispensed in a 96-well plate (1000 cells/well). The 
cells had been pre-incubated in a $5 \%$ carbon dioxide incubator at $37^{\circ} \mathrm{C}$ for 24 hours. The cell culture media has been described previously and contained 100 $\mu \mathrm{L}$ of Biodentine and MTA in the appropriate wells. Periodontal cells treated with $3 \%$ hydrogen peroxide served as positive control and periodontal cells cultured with a-MEM supplemented with $10 \%$ FBS served as negative control. Then, the cells were incubated for 24,48 or 72 hours. Next, $10 \mu \mathrm{L}$ of the CCK-8 solution (Dojindo, Japan) were added to each well and incubated with the cells for 4 hours. Finally, the absorbance was measured at $450 \mathrm{~nm}$. The relative growth rate (RGR) was calculated using the flowing formula:

\section{Micro-Computed Tomography Analysis of the Sealing Ability}

Twenty-one extracted human teeth with a single canal were collected from the outpatient clinic of the Oral Surgery Department at Guanghua School of Stomatology, Sun Yat-sen University. The research was approved by the Ethics Committee of Guanghua School of Stomatology, Sun Yat-sen University (Approval Number ERC-2014-12). All teeth included in this study met the following criteria: a single root canal, mature apices, a lack of severe apical curvatures, and the absence of obvious fractures or cracks.

First, the crown was removed using a water-cooled diamond disc at a distance of $15 \mathrm{~mm}$ from the apex of the root. Then, the apical end was determined, and the standard crown-down technique was used to prepare the canal with a rotary ProTaper system (Dentsply Maillefer, Ballaigues, Switzerland). During instrumentation, irrigation was achieved with $5 \mathrm{~mL}$ of a $17 \%$ EDTA and $5.25 \% \mathrm{NaOCl}$ solution (Sultan Healthcare Inc., Englewood, USA). After preparing the root canal, $5 \mathrm{~mL}$ of a $17 \%$ EDTA and $5.25 \% \mathrm{NaOCl}$ solution were used to rinse the root canal. Finally, gutta-percha (Dentsply De Trey, Konstanz, Germany) and AH Plus (Dentsply De Trey, Konstanz, Germany) were used to complete the root obturation with the warm vertical compaction technique. The root orifices were filled with glass ion. Then, an area located 3 $\mathrm{mm}$ from the apex of all teeth was resected using a diamond burr. Root-ending cavities 3-mm deep and 1.0 $\mathrm{mm}$ in diameter were prepared using an ultrasonic tip (SATELEC, France).

The 21 teeth were divided into three groups: the Biodentine group ( $n=7)$, the MTA group ( $n=7)$, and the blank control group $(n=7)$. Teeth in the blank control group were not prepared or filled. Root- ending cavities were filled with Biodentine and MTA, accordingly. All teeth were incubated at $37^{\circ} \mathrm{C}$ for 7 days. Nail varnish was used to coat the teeth's apical surface to determine microleakage. All teeth were placed in a $50 \%$ silver nitrate solution (Guangzhou Chemical Reagent Factory, China) at room temperature for 12 hours.

The volume of the silver nitrate solution that infiltrated the root canal in all experimental groups was evaluated using micro-computed tomography (microCT) (Bruker micro-CT, Kontich, Belgium). Scanning parameters were $90 \mathrm{kV}, 90 \mu \mathrm{A}$, and $708 \mathrm{~ms}$ with a voxel size of $18 \mu \mathrm{m}$. The scan data were obtained from all groups under the same conditions. Apical leakage was determined by measuring the amount of the silver nitrate solution that had infiltrated into the root canal. The data were analyzed using an image analysis software (VGStudio MAX 1.2.1, Heidelberg, Germany). The silver nitrate solution was recognized and then outlined according to the gray value of the image in each section. Working selections were reconstructed into 3-dimensional images using VGStudio MAX 1.2.1. The silver nitrate solution volumes and depth that infiltrated the root canal were calculated to assess the apical leakage in the MTA and Biodentine groups. All measurements were performed by two calibrated examiners in two different sessions. The interclass correlation coefficient (ICC) between two examiners was greater than 0.8. The ICC between different sessions by the same examiners was greater than 0.9.

Periradicular Surgery and Sample Analysis

This study was approved by the Animal Care and Use Committees of Sun Yat-sen University (IACUCDB-15-1004). The first, second and third maxillary premolars and the first, second, third, and fourth mandibular permanent premolars of 3 female beagle dogs aged 18 months weighing an average of 10-11 $\mathrm{kg}$ were selected for the study ( 72 roots in total). The roots were randomly distributed into 3 groups: MTA (30 roots), Biodentine (30 roots), and negative control (12 roots) groups. Root-end cavities were not prepared or filled in the negative control group.

The animals were anesthetized via intramuscular injections of $3 \%$ pentobarbital $(1 \mathrm{~mL} / \mathrm{kg}$ ). X-rays (60 $\mathrm{kV}, 7 \mathrm{~mA}, 0.2 \mathrm{~s}$ ) were obtained to evaluate the canal system before the surgery. Root canal treatment was performed on each tooth. Briefly, when the experimental teeth were exposed, coronal access was established and the pulp tissue was removed. Initially, 
a $10 \# \mathrm{~K}$ file was used to reach the working length. Next, the ProTaper nickel-titanium system was used to prepare the root canal using an F4 file. During this procedure, saline and 3\% hydrogen peroxide were used to rinse the root canal. The entire canal was filled with gutta-percha supplemented with AH Plus, and the root orifices were sealed with glass ion. X-rays were used to assess the root canal filling.

The procedures used for periradicular surgery were performed according to the method described by Apaydin. ${ }^{18}$ Briefly, a full-thickness mucoperiosteal buccal flap with two releasing incisions was created. The bone from the buccal side was removed with a round bar, and the bone defects were shaped with a high-speed handpiece with saline irrigation; the procedure stopped when the diameter of the defects reached $4 \mathrm{~mm}$. The root ends were resected in all groups with a fissure burr approximately $3 \mathrm{~mm}$ from the apex. A root-ending cavity with a depth of $3 \mathrm{~mm}$ was created with an ultrasonic unit and filled with MTA or Biodentine according to the manufacturer's recommendations. The mucoperiosteal flaps were sutured with 4-0 silk sutures. After surgery, the animals were placed on a soft diet. After six months, the animals were euthanized without pain.

All experimental roots were dissected and scanned using micro-CT. Scanning parameters were 70 kV, 200 $\mu \mathrm{A}$, and $300 \mathrm{~ms}$ with a voxel size of $34.4 \mu \mathrm{m}$. The data were analyzed using VGStudio MAX 1.2.1 software. The bone defects were recognized and labeled according to the gray value of the image of each section. Working selections were reconstructed into 3-dimensional images using VGStudio MAX 1.2.1. Bone defect volumes were calculated to assess the apical healing in the MTA and Biodentine groups. All measurements were performed by two calibrated examiners in two different sessions.

\section{Statistical Analyses}

Statistical analyses were performed using one-way analysis of variance followed by the Student-NewmanKeuls test with SPSS 13.0 software. 95\% confidence intervals were determined. A $P$-value less than 0.05 was considered statistically significant.

\section{Results}

\section{Toxicity of the Materials}

The RGR was set to $100 \%$ in the NC group. At 24 hours, a lower RGR was observed for PDLCs in the MTA group than in the negative control group (NC) $(P<0.01)$, whereas no significant difference was observed between the Biodentine group and the NC group ( $P>0.05$ ). After 48 hours, the RGR of PDLCs in both the MTA and Biodentine groups was lower than the NC group $(P<0.01)$, but no significant differences were observed between the two experimental groups $(P>0.05)$. After 72 hours, no significant differences were observed among the MTA, Biodentine and NC groups (Figure 1).

\section{Determination of Microleakage Using a Micro- CT Analysis}

Based on the quantitative analysis (Figure 2A), a larger volume of the infiltrated nitrate solution was observed in the MTA group than in the Biodentine group (Figure 2B, $P=0.014$ ). The infiltration depth was also greater in the MTA group than in the Biodentine group (Figure 2C, $P=0.005$ ). Both groups showed less apical microleakage than the control group, according to both depth and volume (Figure $2 \mathrm{C}, P<0.001$ ).

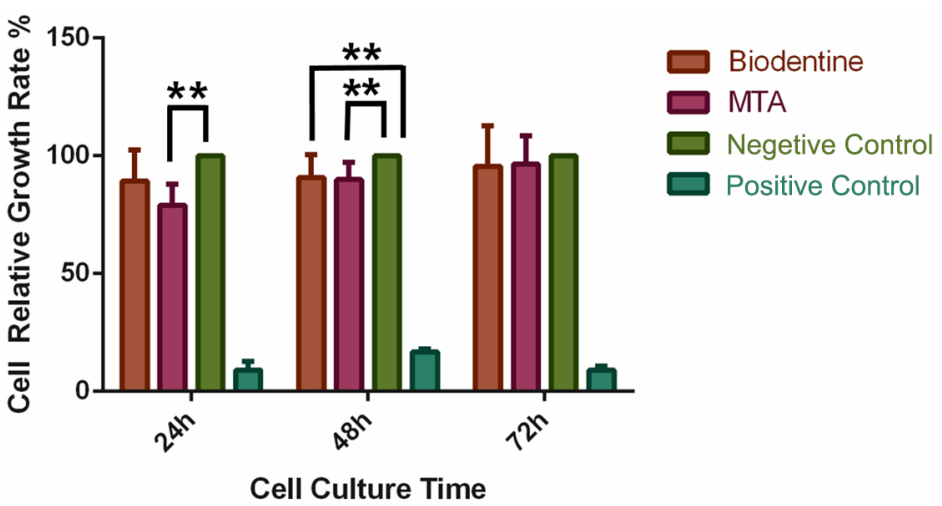

Figure 1- Effects of MTA and BD on human periodontal ligament cells (hPDLCs). hPDLCs were cultured with a-MEM containing MTA or $\mathrm{BD}$ in a 96 -well plate for 24,48 , or 72 hours. The relative growth rate (RGR) was evaluated using a CCK-8 assay. The RGR of the negative control (NC) group was set to $100 \%$, and other groups were compared to the NC group. ${ }^{\star \star} \mathrm{P}<0.01$. 
A

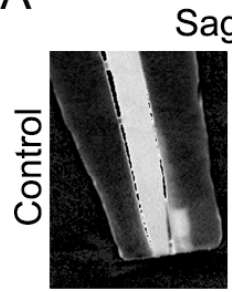

Sagittal

Coronal

$3 \mathrm{D}$
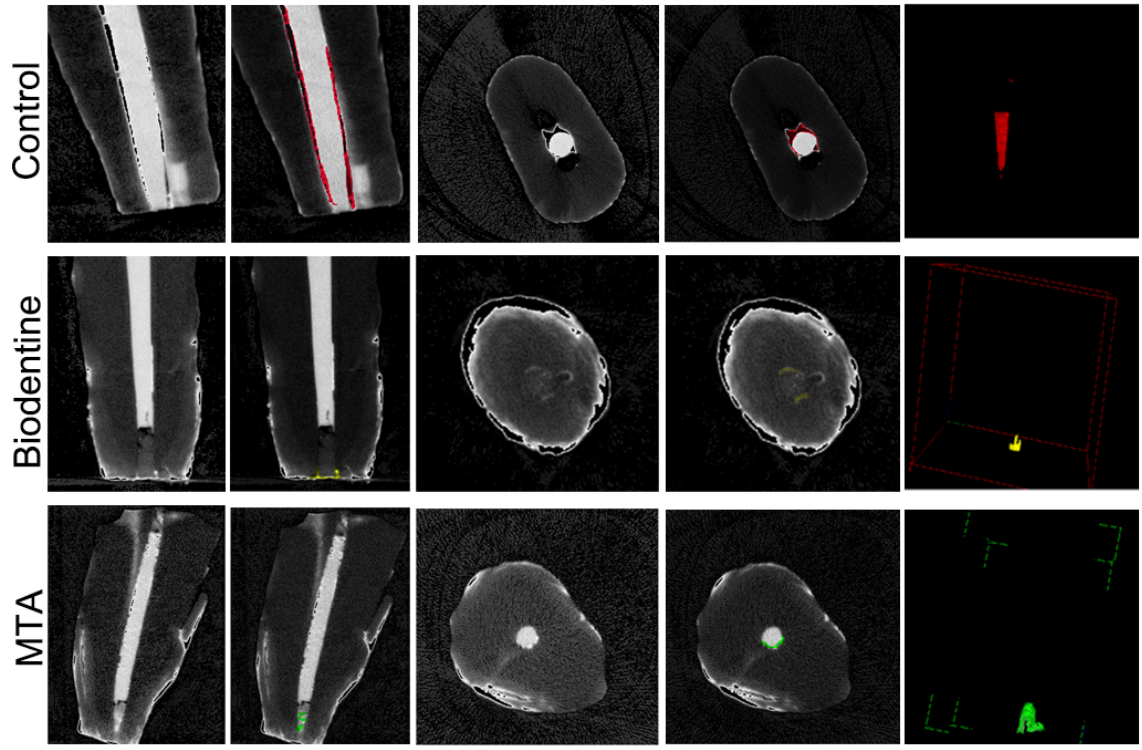

B

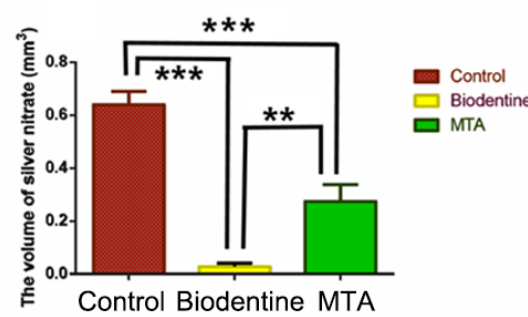

C

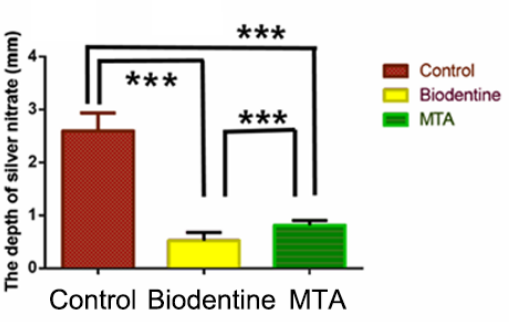

Figure 2- Determination of microleakage using a micro-CT analysis. (A) Micro-CT images showing the volume of the silver nitrate solution that infiltrated the root canal in each experimental group. (B) Quantitative analysis of the volume of the nitrate solution that infiltrated the root canal. (C) Quantitative analysis of the depth of the nitrate solution that infiltrated the root canal. ${ }^{* *} \mathrm{P}<0.01$ and ${ }^{* * *} \mathrm{P}<0.001$.

\section{Evaluation of Bone Formation Using Micro-CT}

The volume of the bone defect in the MTA group was larger than in the Biodentine group. However, the difference observed between the MTA and Biodentine groups was not significant $(P>0.05)$. The volumes of the bone defects in the MTA and Biodentine groups were both smaller than the defects in the NC group $(P<0.05)$. The statistical evaluation of tissue formation at six months after surgery indicated that both MTA and Biodentine promoted bone tissue regeneration (Figure 3).

\section{Discussion}

In the present study, Biodentine possessed a similar sealing ability and biocompatibility to MTA. Thus, Biodentine has great potential as an alternative to MTA in periradicular surgery.

According to the results of the CCK- 8 assay, both MTA and Biodentine exhibited low levels of cytotoxicity at 48 hours, but not at 72 hours. The low levels of cytotoxicity induced by both materials in the early stage can be due to a hydration reaction. MTA and Biodentine contain calcium silicate, so calcium hydroxide would be created in a hydration reaction when calcium silicate reacts with water. Calcium hydroxide can increase the $\mathrm{pH}$ of the culture media, which damages PDSCs. Other biocompatibility studies showed that MTA and Biodentine exhibited slight cytotoxicity, but the toxic effects were less than in other materials, such as octacalcium phosphate and IRM. ${ }^{19-21}$

Sealing ability is a key characteristic of root-end materials that determines the success of periapical surgery. The common methodologies used to assess apical and coronal leakage include the dye penetration and extraction method, the fluid filtration or transportation method, and the bacteria and toxin infiltration method. 22 Due to its low cost and simplicity, the dye penetration and extraction method has been the technique preferred by most researchers. However, the teeth must be sectioned longitudinally or transversely to examine the extent of dye penetration. With this technique, the sample is destroyed and only 
A
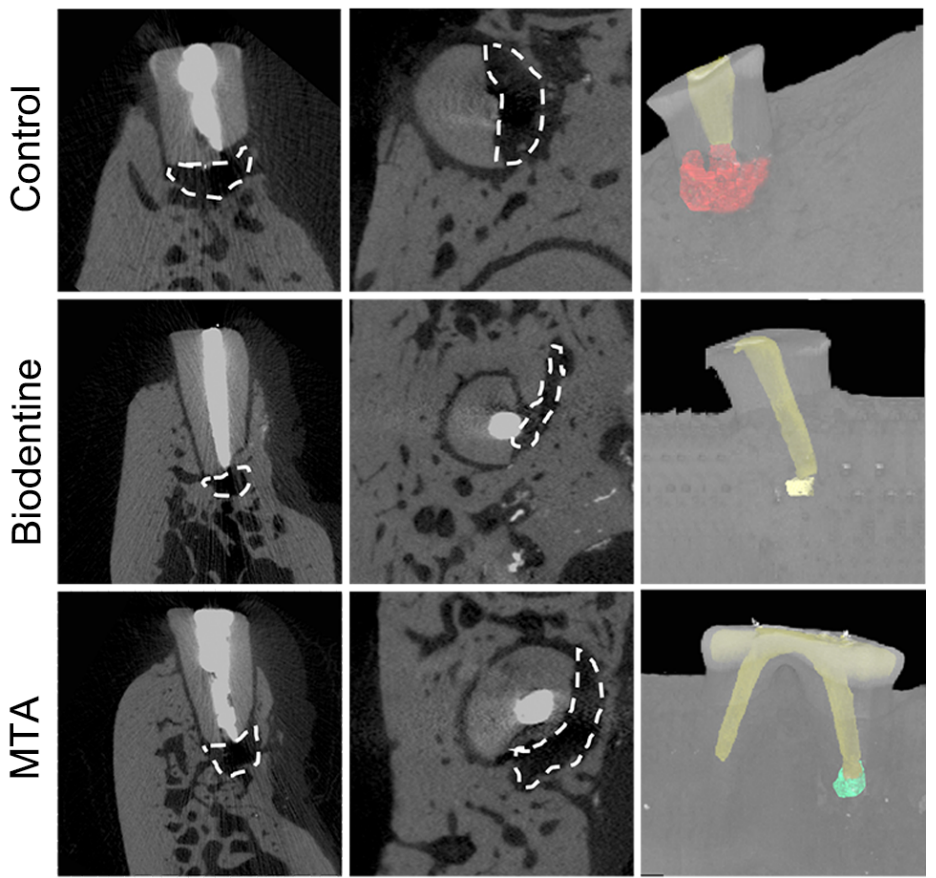

B

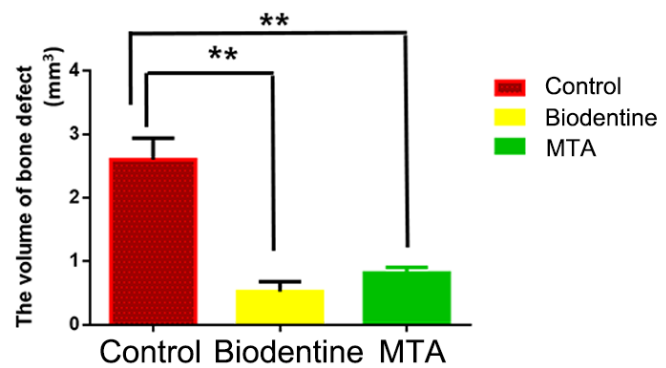

Figure 3- Evaluation of bone formation using micro-CT after six months of root-end filling of dogs' teeth. (A) Volume of bone defects in the negative control group, BD root-end filling and MTA root-end filling groups. (B) Quantitative analysis of bone defect volumes in each group. ${ }^{* *} \mathrm{P}<0.01$

the sectioned planes are evaluated. ${ }^{23}$ In previous studies, researchers obtained three 1 -mm thick transverse sections by sectioning the apical portions of the roots, and then used confocal laser scanning microscopy (CLSM) to determine gaps between the root-end filling materials and the dentin. ${ }^{24}$ In these experiments, the results only reflected the twodimensional gaps. In addition, because transverse sections and the selected areas measured with CLSM are limited, the results were inevitably affected by subjective factors.

Interestingly, a study by Kakaboura, et al. ${ }^{25}$ (2007) evaluated the 3D-marginal adaptation between dentine and resin composites using computerized $X$-ray microtomography. This method possesses unique advantages such as full 3D-fidelity, enabling a quantitative evaluation of the interfacial adaptation at any site and direction. ${ }^{26}$ We evaluated the microleakage between dentine and root-end filling materials with a similar method described by Kakaboura, et al. ${ }^{25}$ (2007).
The entire leakage volume and depth were imaged, with no destruction of the samples. In the present study, a greater volume and depth of the infiltrated nitrate solution was observed in the MTA group than in the Biodentine group. However, according to Soundappan, et al. ${ }^{27}$ (2014), the marginal adaptation of MTA and IRM was superior to Biodentine when used as root-end filling materials. These divergent results may be attributed to the differences in the accuracy of these methods. We measured microleakage with 3D imaging, which was more accurate than a simple qualitative evaluation of microleakage in transverse sections. Based on our 3D evaluation, we conclude that the sealing ability of Biodentine is adequate when applied in root-end fillings. The limitation of this study is that we only scanned the teeth after immersion. The results might be more convincing if we obtain the scan data before immersion in silver nitrate solution and compare them with the scanned data after immersion.

Furthermore, we explored the biological effect of 
Biodentine in vivo. Although the beagle dog is more expensive and difficult to operate upon, it is still more suitable than other animals, such as mice and rats, as a model of the apical responses in humans. In addition, the teeth of other small animals are too small to operate on using the root-end filling technique. Hence, we chose beagle dogs as a periradicular surgery model. The design of the animal experiment used in the present study differs from other similar studies because apical periodontitis was not induced before treatment. The aim of this design was to eliminate the confounding effect of induced periodontitis on assessing the biocompatibility of Biodentine. For instance, when treatment failure occurs in a tooth with induced periodontitis, a determination of whether the cause of failure was attributed to the material would be difficult. ${ }^{28}$

The beagle dog model has been widely used to evaluate the characteristics of dental materials, including Biodentine. For example, Silva, et al. ${ }^{15}$ (2017). showed that Biodentine can be used as a furcation perforation repair material in the beagle dog model, with comparable results to MTA. According to De Rossi et al. ${ }^{29}$ (2014), Biodentine and MTA both facilitate mineralized tissue bridge formation after pulpotomy. However, no study has compared Biodentine and MTA when applied as root-end filling materials in vivo. In the present beagle dog periradicular surgery model, micro-CT was used to evaluate the bone healing process and the results showed that both materials exhibited good effects on periradicular bone healing. As shown in the study by Daltoe, et al. ${ }^{14}$ (2016), the ability of Biodentine to upregulate mineralization markers was similar to MTA. In addition, $\mathrm{Ho}$, et al. ${ }^{30}$ (2018) confirmed the potential utility of Biodentine in dental and bone regeneration using threedimensional printed Biodentine/polycaprolactone composite scaffolds. These studies have confirmed that Biodentine promotes bone healing.

\section{Conclusions}

Biodentine and MTA showed comparable cell biocompatibility. Biodentine was superior to MTA in terms of sealing ability when used as a root-end filling material. Both Biodentine and MTA promoted periradicular bone healing after periradicular surgery in beagle dog models.

\section{Conflict of Interest}

The authors have no conflicts of interest to declare.

\section{Ethical Approval}

All applicable international, national, and/or institutional guidelines for the care and use of animals were followed. All procedures involving human participants were performed in accordance with the ethical standards of the institutional and/or national research committee and with the 1964 Declaration of Helsinki and its later amendments or comparable ethical standards.

\section{Informed Consent}

Informed consent was obtained from all individual participants included in the study.

\section{Acknowledgments}

This study was supported by grants from (1) the International Cooperation Project of Science and Technology in Guangdong Province (2016B050502008); (2) the National Natural Science Foundation of China (Grant No. 81670984, No. 81873713). The authors indicate no potential conflicts of interest.

\section{References}

1- Kohli M, Berenji H, Setzer F, Lee S, Karabucak B. Outcome of endodontic surgery: a meta-analysis of the literature-part 3: comparison of endodontic microsurgical techniques with 2 different root-end filling materials. J Endod. 2018;44(6):923-31. doi: 10.1016/j. joen.2018.02.021

2- Kim S, Kratchman S. Modern endodontic surgery concepts and practice: a review. J Endod. 2006;32(7):601-23. doi: 10.1016/j. joen.2005.12.010

3- Parirokh M, Torabinejad M. Mineral trioxide aggregate: a comprehensive literature review - Part I: chemical, physical, and antibacterial properties. J Endod. 2010;36(1):16-27. doi: 10.1016/j. joen.2009.09.006

4- Shipper G, Grossman E, Botha A, Cleaton-Jones P. Margina adaptation of mineral trioxide aggregate (MTA) compared with amalgam as a root-end filling material: a low-vacuum (LV) versus high-vacuum (HV) SEM study. Int Endod J. 2004;37(5):325-36. doi: 10.1111/j.01432885.2004.00806.x

5- Parirokh M, Torabinejad M. Mineral trioxide aggregate: a comprehensive literature review-Part III: clinical applications, drawbacks, and mechanism of action. J Endod. 2010;36(3):400-13. doi: 10.1016/j.joen.2009.09.009.

6- Watts JD, Holt DM, Beeson TJ, Kirkpatrick TC, Rutledge RE. Effects of $\mathrm{pH}$ and mixing agents on the temporal setting of tooth-colored and gray mineral trioxide aggregate. J Endod. 2007;33(8):970-3. doi: 10.1016/j.joen.2007.01.024. 
7- Sousa Reis M, Scarparo RK, Steier L, Figueiredo JA. Periradicular inflammatory response, bone resorption, and cementum repair after sealing of furcation perforation with mineral trioxide aggregate (MTA Angelus) or Biodentine. Clin Oral Investig. Forthcoming 2019. doi: 10.1007/s00784-019-02833-z

8- Parinyaprom N, Nirunsittirat A, Chuveera P, Na Lampang S, Srisuwan T, Sastraruji T, et al. Outcomes of direct pulp capping by using either proRoot mineral trioxide aggregate or Biodentine in permanent teeth with carious pulp exposure in 6- to 18-year-old patients: a randomized controlled trial. J Endod. 2018;44(3):341-8. doi: 10.1016/j.joen.2017.10.012.

9- Lipski M, Nowicka A, Kot K, Postek-Stefanska L, WysoczanskaJankowicz I, Borkowski $L$, et al. Factors affecting the outcomes of direct pulp capping using Biodentine. Clin Oral Investig. 2018;22(5):2021-9. doi: $10.1007 /$ s00784-017-2296-7

10- Brizuela C, Ormeno A, Cabrera C, Cabezas R, Silva CI, Ramirez $\mathrm{V}$, et al. Direct Pulp capping with calcium hydroxide, mineral trioxide aggregate, and Biodentine in permanent young teeth with caries: a randomized clinical trial. J Endod. 2017;43(11):1776-80. doi: 10.1016/j.joen.2017.06.031

11- Katge FA, Patil DP. Comparative analysis of 2 calcium silicatebased cements (Biodentine and Mineral Trioxide Aggregate) as direct pulp-capping agent in young permanent molars: a split mouth study. J Endod. 2017;43(4):507-13. doi: 10.1016/j.joen.2016.11.026

12- Sharma S, Sharma V, Passi D, Srivastava D, Grover S, Dutta SR. Large periapical or cystic lesions in association with roots having open apices managed nonsurgically using 1-step apexification based on platelet-rich fibrin matrix and biodentine apical barrier: a case series. J Endod. 2018;44(1):179-85. doi: 10.1016/j.joen.2017.08.036.

13-Vidal K, Martin G, Lozano O, Salas M, Trigueros J, Aguilar G. Apical closure in apexification: a review and case report of apexification treatment of an immature permanent tooth with biodentine. J Endod. 2016;42(5):730-4. doi: 10.1016/j.joen.2016.02.007

14- Daltoe MO, Paula-Silva FW, Faccioli LH, Gaton-Hernandez PM, De Rossi A, Bezerra Silva LA. Expression of mineralization markers during pulp response to Biodentine and Mineral Trioxide Aggregate. J Endod. 2016;42(4):596-603. doi: 10.1016/j.joen.2015.12.018

15- Silva LAB, Pieroni K, Nelson-Filho P, Silva RAB, Hernandez-Gaton P, Lucisano MP, et al. Furcation perforation: periradicular tissue response to biodentine as a repair material by histopathologic and indirect immunofluorescence analyses. J Endod. 2017;43(7):1137-42. doi: 10.1016/j.joen.2017.02.001

16- Vajrabhaya LO, Korsuwannawong S, Jantarat J, Korre S. Biocompatibility of furcal perforation repair material using cell culture technique: ketac molar versus ProRoot MTA. Oral Surg Oral Med Oral Pathol Oral Radiol Endod. 2006 Dec;102(6):e48-50. doi: 10.1016/j. tripleo.2006.05.015

17- Vidovic Zdrilic I, Azevedo Queiroz IO, Matthews BG, Gomes-Filho JE, Mina M, Kalajzic I. Mineral trioxide aggregate improves healing response of periodontal tissue to injury in mice. J Periodontal Res. 2017;52(6):1058-67. doi: 10.1111/jre.12478
18- Apaydin ES, Shabahang S, Torabinejad M. Hard-tissue healing after application of fresh or set MTA as root-end-filling material. J Endod. 2004;30(1):21-4. 10.1097/00004770-200401000-00004

19- Luo T, Liu J, Sun Y, Shen Y, Zou L. Cytocompatibility of Biodentine and iRoot FS with human periodontal ligament cells: an in vitro study. Int Endod J. 2018;51(7):779-88.

20- Tomas-Catala CJ, Collado-Gonzalez M, Garcia-Bernal D, OnateSanchez RE, Forner L, Llena $C$, et al. Biocompatibility of new pulpcapping materials NeoMTA plus, MTA Repair HP, and Biodentine on human dental pulp stem cells. J Endod. 2018;44(1):126-32. doi: 10.1016/j.joen.2017.07.017

21- Min KS, Chang HS, Bae JM, Park SH, Hong CU, Kim EC. The induction of heme oxygenase-1 modulates bismuth oxide-induced cytotoxicity in human dental pulp cells. J Endod. 2007;33(11):1342-6. 10.1016/j.joen.2007.07.012

22- Verissimo DM, Vale MS. Methodologies for assessment of apical and coronal leakage of endodontic filling materials: a critical review. J Oral Sci. 2006;48(3):93-8.

23- Orosco F, Bramante C, Garcia R, Bernardineli N, Moraes I. Sealing ability, marginal adaptation and their correlation using three root-end filling materials as apical plugs. J Appl Oral Sci. 2010;18(2):127-34. 24- Ravichandra PV, Vemisetty H, Deepthi K, Jayaprada Reddy S, Ramkiran D, Jaya Nagendra Krishna $M$, et al. Comparative evaluation of marginal adaptation of biodentine(TM) and other commonly used root end filling materials - an in vitro study. J Clin Diagn Res. 2014;8(3):2435. 10.7860/JCDR/2014/7834.4174

25- Kakaboura A, Rahiotis C, Watts D, Silikas N, Eliades G. 3D-marginal adaptation versus setting shrinkage in light-cured microhybrid resin composites. Dent Mater. 2007;23(3):272-8. doi: 10.1016/j. dental.2006.01.020

26- Sun J, Eidelman N, Lin-Gibson S. 3D mapping of polymerization shrinkage using $\mathrm{X}$-ray micro-computed tomography to predict microleakage. Dent Mater. 2009;25(3):314-20. doi: 10.1016/j. dental.2008.07.010

27- Soundappan S, Sundaramurthy JL, Raghu S, Natanasabapathy V. Biodentine versus mineral trioxide aggregate versus intermediate restorative material for retrograde Root end filling: an in vitro study. J Dent (Tehran). 2014;11(2):143-9.

28- Kohout GD, He J, Primus CM, Opperman LA, Woodmansey KF. Comparison of quick-set and mineral trioxide aggregate root-end fillings for the regeneration of apical tissues in dogs. J Endod. 2015;41(2):24852. Doi: 10.1016/j.joen.2014.10.005

29- De Rossi A, Silva LA, Gaton-Hernandez P, Sousa-Neto MD, NelsonFilho $\mathrm{P}$, Silva RA, et al. Comparison of pulpal responses to pulpotomy and pulp capping with biodentine and mineral trioxide aggregate in dogs. J Endod. 2014;40(9):1362-9. doi: 10.1016/j.joen.2014.02.006 30- Ho CC, Fang HY, Wang B, Huang TH, Shie MY. The effects of Biodentine/polycaprolactone three-dimensional-scaffold with odontogenesis properties on human dental pulp cells. Int Endod J. 2018;51 Suppl 4:e291-e300. doi:10.1111/iej.12799 